\title{
Functionalization of Mesoporous Silicon Nanoparticles for Targeting and Bioimaging Purposes
}

\author{
Jussi Rytkönen, ${ }^{1,2}$ Riitta Miettinen, ${ }^{3,4}$ Martti Kaasalainen, ${ }^{5}$ Vesa-Pekka Lehto, ${ }^{6}$ \\ Jarno Salonen, ${ }^{5}$ and Ale Närvänen ${ }^{1,2}$ \\ ${ }^{1}$ School of Pharmacy, University of Eastern Finland, P.O. Box 1627, 70211 Kuopio, Finland \\ ${ }^{2}$ Biocenter Kuopio, 70211 Kuopio, Finland \\ ${ }^{3}$ Department of Neurology, University of Eastern Finland, P.O. Box 1627, 70211 Kuopio, Finland \\ ${ }^{4}$ CN Services Ltd., Microkatu 1, 70210 Kuopio, Finland \\ ${ }^{5}$ Department of Physics and Astronomy, University of Turku, Vesilinnantie 5, 20014 Turku, Finland \\ ${ }^{6}$ Department of Applied Physics, University of Eastern Finland, P.O. Box 1627, 70211 Kuopio, Finland
}

Correspondence should be addressed to Ale Närvänen, ale.narvanen@uef.fi

Received 5 April 2012; Accepted 3 June 2012

Academic Editor: Renyun Zhang

Copyright ( 2012 Jussi Rytkönen et al. This is an open access article distributed under the Creative Commons Attribution License, which permits unrestricted use, distribution, and reproduction in any medium, provided the original work is properly cited.

\begin{abstract}
Carboxylic acid functionalized thermally hydrocarbonized porous silicon nanoparticles (UnTHCPSi-NP) were synthesized, and their opsonization and targeting properties were studied in vitro alongside with in vivo biodistribution. The carboxyl groups on UnTHCPSi were utilized to further functionalize the nanoparticles. In order to reduce the opsonization of the UnTHCPSiNPs, different sized polyethylene glycol (PEG) were conjugated or adsorbed to the NPs surface. The latter approach, based on hydrophobic interaction, turned out to be more effective in reducing the opsonization and improving the stability of the nanoparticle suspension. The most abundant opsonins after plasma incubation were fibrinogen precursors and IgG. Furthermore, the targeting properties of UnTHCPSi-NPs were studied in vitro with antibodies against glutathione S-transferase (anti-GST). PEGylated NPs conjugated with anti-GST bound to GST-agarose in human plasma nearly 35-fold compared to control NPs, indicating that UnTHCPSi-NPs are suitable for targeting in physiological environment. The in vivo biodistribution in mice revealed that PEGylated UnTHCPSi-NPs, accumulate fast into the liver and the spleen, regardless of the reduced opsonization in vitro. However, autoradiography and transmission electron microscopy showed that majority of the NPs still remained in hepatic blood vessels and sinusoids suggesting a possibility to utilize them as a sustained release platform for payload molecules.
\end{abstract}

\section{Introduction}

Utilization of nanoparticles (NPs) is currently emerging also in the field of drug delivery. Formulation of the drug molecules with nanoparticles modulates their pharmacokinetics by several means: by modifying the dissolution and absorption rates, by protection the molecules from enzymatic and chemical degradation, and by enabling preferential accumulation in the desired targets. Mesoporous silicon (PSi) micro- and nanoparticles are promising drug carriers for the targeted therapy, for example, due to their high payload capacity of therapeutic agents and biocompatibility $[1,2]$. Depending on the size and the surface chemistry of the pores, increased or sustained release of the loaded therapeutic agents can be obtained [2,3].
Intravenous drug delivery is an interesting approach to cancer treatment. The drawback of this strategy is that intravenously administered particulates are known to be rapidly removed from the blood stream by macrophages of the mononuclear phagocyte system (MPS) [4]. Macrophages do not directly recognize NPs, but they recognize the plasma proteins, for example, opsonins that bind on the surface of the NPs [5]. Agglomeration of NPs in physiological buffers or in plasma and the fast clearance to liver and spleen by MPS may restrict their use as effective drug carriers [4]. The sensitivity of the MPS towards administered NPs is depending on the size, shape, surface charge, and surface chemistry of the NPs beside the structure and composition of the NPs $[4,6-8]$. Due to the strong negative or positive charge, serum proteins bind to the NPs causing fast clearance 
from the blood stream [9]. On the other hand, the particles with low surface charges show poor dispersion stability and are more susceptible for agglomeration, due to lack of electric repulsion [10].

Several polymers have been used to shield NPs [11]. The most used molecule for the surface shielding is polyethylene glycol (PEG), which is an inert hydrophilic polymer and is invisible to the immunological response. It has been shown that PEGylation decreases the MPS uptake and increases the circulation times of the various types of NPs $[12,13]$. Targeted drug delivery can reduce the systemic exposure of the drug and minimize its side-effects. This is possible by conjugating targeting moieties to the NPs surface. Several types of ligands have been utilized in targeted NPs, including antibodies, peptides, and aptamers [14, 15]. Especially cancer-specific antibodies have been shown to accumulate to the tumor tissue and they have been used as therapeutic and imaging agents [16]. Antibody conjugated paramagnetic nanoparticles have shown to be effective imaging agents in magnetic resonance (MRI) tumor imaging [17].

In the present study, we describe the production of thermally carbonized porous silicon nanoparticles derivatized with carboxylic acid (UnTCPSi-NP). Furthermore, these NPs were functionalized with PEG, antibodies and radiotracer, which were covalently conjugated on the surface UnTHCPSiNPs via the carboxyl groups. Consequently, we studied the opsonization and targeting properties of the functionalized UnTHCPSi-NPs in vitro. The opsonization studies were conducted with two different PEGylation methods and varying PEG length. The opsonins were identified from NPs incubated in human plasma with gel electrophoresis and mass spectrometer. In addition, the targeting properties of UnTHCPSi-NPs were tested with in vitro immunoprecipitation. Finally, the biodistribution of PEGylated NPs was tested in healthy mice. Autoradiography and transmission electron microscopy (TEM) were subsequently used to study the distribution and subcellular location of the nanoparticles from the tissue samples.

\section{Materials and Methods}

2.1. Nanoparticle Production. Mesoporous silicon (PSi) nanoparticles were fabricated with electrochemical etching of p+-type single crystal silicon wafers with resistivity values of $0.01-0.02 \Omega \mathrm{cm}$. HF (38\%)-ethanol mixture was used as an electrolyte. Multilayered films were fabricated with pulse etching method by applying two different current densities successively. Lower etching current of $50 \mathrm{~mA} / \mathrm{cm}^{2}$ was applied in order to form mesoporous layers which were separated by high porosity fracture layer etched with higher $200 \mathrm{~mA} / \mathrm{cm}^{2}$ current density [18]. Multilayered PSi film was lifted-off by using high etching current at electropolishing region. After anodization, multilayered PSi film was thermally hydrocarbonized (THCPSi) under continuous $\mathrm{N} 2 /$ acetylene $(1: 1)$ flow at $500^{\circ} \mathrm{C}$. The treatment enables partial hydrocarbon termination of the surface thus making it hydrophopic $[18,19]$. Functionalization of THCPSi-film surface was made by thermal treatment in undecylenic acid as described in detail by Kovalainen et al. [20].

After the surface treatments, the PSi films were wet ballmilled with fritsch pulverisette 7 and ethanol was used as a grinding medium. Approximately $1 \mathrm{~g}$ of sample and $20 \mathrm{~mL}$ of ethanol was used while ball-to-sample ratio was $60: 1$, which has been found to be enough to fracture porous silicon films to nanoparticles [21]. Milling was carried out at $800 \mathrm{rpm}$ and milling-time was $3 \mathrm{~h}$. After milling, ethanol was changed to DMF by centrifuging. Finally the UnTHCPSi-NPs were separated from polydisperse milling-mixture by centrifuging it with relative acceleration of $1700 \mathrm{~g}$.

2.2. Oleyl-PEG Synthesis. Oleyl-PEG (O-PEG) was synthesized by adapting the previously described work by Silvander et al. [22]. Briefly, $0.40 \mathrm{~g}$ of $\mathrm{H}_{2} \mathrm{~N}-\mathrm{PEG}-\mathrm{OMe}$ (IRIS Biotech) with different molecular weights $(5 \mathrm{kDa}, 10 \mathrm{kDa}$, or $20 \mathrm{kDa})$, was mixed with $1.5 \mathrm{mmoL}$ oleyl-chloride (Sigma-Aldrich), in $15 \mathrm{~mL}$ of dry $\mathrm{CHCl}_{3}$ containing $1.5 \mathrm{mmoL}$ triethylamine (Fluka). The reaction mixture was stirred $18 \mathrm{~h}$ at room temperature (RT) under nitrogen atmosphere. The product was purified by dissolving the crude product in acetone and precipitating with diethylether. Precipitation was repeated twice. The product was confirmed with thin layer chromatography (iodine and ninhydrin staining) and ${ }^{1} \mathrm{H}$ NMR analysis.

2.3. Carbodiimide Activation of the Carboxyl Groups on UnTHCPSi-NP Surface. The carboxyl groups on the surface of the UnTHCPSi-NPs were activated with $\mathrm{N}, \mathrm{N}^{\prime}$ diisopropylcarbodiimide (DIC, PerSeptive Biosystems $\mathrm{GmbH}$ ) and N-hydroxysuccinimide (NHS, Fluka). The UnTHCPSi-NPs (1 mg), suspended in dimethyl formamide (DMF), were activated with $40 \mathrm{mM}$ of DIC and NHS in $1 \mathrm{~mL}$ of DMF by mixing for 1 hour at RT. Excess of the activation reagents were removed by centrifugation, 20 minutes $13200 \mathrm{rpm}$ (Eppendorf Centrifuge 5425, Eppendorf $A G)$. The supernatant was removed and the UnTHCPSi-NPs were washed three times with $1 \mathrm{~mL}$ of acetonitrile (ACN) by using centrifugation as described above. After final washing the UnTHCPSi-NPs were dispersed into small amount of $\mathrm{ACN}$ and stored for further use at $-20^{\circ} \mathrm{C}$.

2.4. Radiolabelling of UnTHCPSi-NPs. $0.2 \mathrm{mg}$ of L-tyrosine (Sigma-Aldrich) $0.3 \mathrm{mg} / \mathrm{mL}$ in water was added onto previously activated UnTHCPSi-NPs, suspended into $333 \mu \mathrm{L}$ of $\mathrm{ACN}$, and mixed for one hour at RT. Excess reagents were removed by centrifugation and washed three times with $1 \mathrm{~mL}$ of $\mathrm{H}_{2} \mathrm{O}$ (Figure 1A). The particles were radiolabelled with iodine-125 via tyrosine residue using Pierce Iodo-Gen precoated iodination tubes (Thermo Scientific). $90 \mu \mathrm{L}$ of $10 \mathrm{mM}$ HEPES (Sigma-Aldrich) pH 7.4 was mixed with $20 \mu \mathrm{L}$ of $\mathrm{Na}^{125} \mathrm{I}$ (6.5 MBq, MDS Nordion S.A. Zeoning Industriel) in $10 \mathrm{mM} \mathrm{NaOH}$ in the Iodo-Gen tube. The reaction was incubated for 6 minutes in room temperature. The oxidized iodine solution was removed from the Iodo-Gen tube and the tyrosinated UnTHCPSi-NPs were suspended into this 


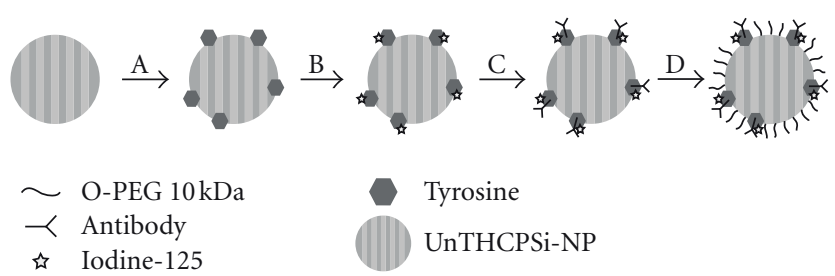

Figure 1: A schematic figure of the surface modifications of UnTHCPSi-NPs. L-tyrosine is covalently conjugated to the carboxylic groups on the UnTHCPsi surface (A). The UnTHCPSiNPs are radiolabelled by incorporating radioactive iodine- 125 to tyrosine residue (B). Antibodies are conjugated to radiolabelled UnTHCPSi-NPs (C). Finally the UnTHCPSi-NPs are PEGylated with O-PEG physisorption (D).

mixture. The particles were incubated for 15 minutes at RT and washed three times with $\mathrm{H}_{2} \mathrm{O}$ (Figure 1B).

2.5. PEGylation of UnTHCPSi-NPs. The UnTHCPSi-NPs were PEGylated either by adsorbing O-PEG or coupling covalently $\mathrm{H}_{2} \mathrm{~N}-\mathrm{PEG}-\mathrm{OMe}$ (C-PEG, IRIS Biotech). For adsorption, $0.5 \mathrm{mg}$ of UnTHCPSi-NPs were suspended into $101 \mu \mathrm{M}$ O-PEG in $1 \mathrm{~mL}$ of water. PEG length was varied $(5 \mathrm{kDa}, 10 \mathrm{kDa}$, and $20 \mathrm{kDa})$. For covalent conjugation, $0.5 \mathrm{mg}$ of previously activated UnTHCPSi-NPs were suspended into $101 \mu \mathrm{M} \mathrm{H} \mathrm{H}_{2} \mathrm{~N}$-PEG-OMe in $1 \mathrm{~mL}$ of $33 \% \mathrm{ACN}$ in $\mathrm{H}_{2} \mathrm{O}$. PEG length was varied ( $5 \mathrm{kDa}, 10 \mathrm{kDa}$, and $20 \mathrm{kDa}$ ). All of the reactions were mixed over night at RT. After the PEGylation, the UnTHCPSi-NPs were washed three times with $\mathrm{H}_{2} \mathrm{O}$ by centrifugation. The average hydrodynamic size and zeta-potential were measured with dynamic light scatter (DLS) and the particle size was confirmed with TEM.

2.6. Conjugation of Antibodies to Radiolabelled UnTHCPSi$N P s$. For the antibody conjugation, $1.0 \mathrm{mg}$ of radiolabelled UnTHCPSi-NPs (non-PEGylated) were dispersed in DMF and activated with DIC and NHS as described above. Antiglutathione S-transferase (a-GST, Rockland) or nonbinding control antibody, human immunoglobulin G (hIgG, Rockland) were conjugated to reactivated radiolabelled UnTHCPSi-NPs in a ratio of $1.29 \mathrm{nmoL}$ of total protein per $1 \mathrm{mg}$ of nanoparticle. Briefly, proteins were dissolved in $800 \mu \mathrm{L}$ of $10 \mathrm{mM}$ HEPES, $\mathrm{pH}$ 7.4. Reactivated and radiolabelled particles, suspended in $200 \mu \mathrm{L}$ ACN, were added into the mixture. The suspension was mixed for $30 \mathrm{~min}$ at RT and washed three times with $\mathrm{H}_{2} \mathrm{O}$ (Figure 1C). Finally the a-GST or hIgG antibody conjugated NPs were PEGylated by adsorption of $10 \mathrm{kDa} \mathrm{O}-\mathrm{PEG}$ as previously mentioned (Figure 1D).

2.7. In Vitro Opsonization Studies. The in vitro opsonization studies were performed with human plasma derived from voluntary people and conducted in similar method as described by Sarparanta et al. [23]. $0.2 \mathrm{mg}$ of UnTHCPSiNPs (PEGylated NPs and non-PEGylated NP as a control) were suspended into $330 \mu \mathrm{L}$ of $50 \%$ human plasma, diluted with phosphate buffered saline (PBS) pH 7.4. The mixtures were incubated at $+37^{\circ} \mathrm{C}$ for 15 minutes. The opsonized UnTHCPSi-NPs were centrifuged at $13200 \mathrm{rpm}$ for $10 \mathrm{~min}$ and washed once with $1 \mathrm{~mL} \mathrm{H}_{2} \mathrm{O}$. Finally the hydrodynamic size of the nanoparticles was measured with DLS.

2.8. DLS Measurements. The nanoparticle samples were diluted to $0.17 \mathrm{mg} / \mathrm{mL}$ with $\mathrm{H}_{2} \mathrm{O}$ prior the measurement. The zeta potential and hydrodynamic size measurements we carried out with Zetasizer Nano-ZS (Malvern instruments, Southborough MA, USA) in $23^{\circ} \mathrm{C}$.

2.9. Protein Analysis of the Opsonins. The proteins were extracted from opsonized UnTHCPSi-NPs (non-PEGylated and $10 \mathrm{kDa}$ O-PEGylated) with sodium dodecyl sulphate polyacrylamide gel electrophoresis (SDS-PAGE). First, $50 \mu \mathrm{g}$ of opsonized UnTHCPSi-NPs suspended in $15 \mu \mathrm{L}$ of PAGE sample buffer (125 mM Tris- $\mathrm{HCl} \mathrm{pH}$ 6.8, 2\% SDS, 5\% glycerol, and $0.002 \%$ bromophenol blue) were incubated for 5 minutes at $100^{\circ} \mathrm{C}$. After the sample preparation the suspensions were applied in SDS-PAGE-gel (12\% acrylamide) and run for 1.5 hours with $100 \mathrm{~V}$ and $400 \mathrm{~mA}$. After the run, the gel was stained with $0.025 \%$ Coomassie brilliant blue (Thermo Scientific). Major protein bands were excised and digested in-gel using trypsin (Fluka) in $+37^{\circ} \mathrm{C}$ at $\mathrm{pH} 8$ for $18 \mathrm{~h}$.

The tryptic peptides were measured with QSTAR XL hybrid quadrupole TOF instrument (Applied Biosystems, Foster City, CA, USA) and the proteins identified from peptide fingerprint data with the MASCOT search v1.6b25 script 27 [24]. The used hardware and protein identification methods have been described in detail previously [25] with the following exceptions. Peptides were separated with the Zorbax 300SB-C18 $(3.5 \mu \mathrm{m} 150 \mathrm{~mm} \times 75 \mu \mathrm{m})$ column (Agilent) at a flow rate of $1 \mu \mathrm{L} / \mathrm{min}$. The peptides were eluted with ACN gradient using 5\% ACN in $0.1 \%$ formic acid as buffer $\mathrm{A}$ and $95 \% \mathrm{ACN}$ in $0.1 \%$ formic acid as buffer $\mathrm{B}$. First 3 minutes of the gradient $100 \%$ buffer A, from 3 to 25 minutes down to $55 \%$ buffer A, from 25 to 35 minutes down to $15 \%$ buffer A. The buffer A was kept at the same level for 45 minutes. The buffer A was brought back to $100 \%$ by 46 minutes. The total run time was 80 minutes. The mass spectrometer instrument was calibrated on known trypsin autolysis peptides. The MASCOT searches were carried out against MSDB 20060831 database, which contained 3239079 sequences. Parent ion and fragment mass tolerances were 0.1 and $0.2 \mathrm{Da}$, respectively. Only oxidation of methionine (M) was selected as a variable modification.

2.10. Biotargeting of UnTHCPSi-NPs with Immunoprecipitation. The biotargeting of the PEGylated, anti-GST-antibody conjugated and radiolabelled UnTHCPSi-NPs was tested in vitro with glutathione S-transferase-(GST-) agarose (Rockland). The GST-agarose was first blocked with 1\% bovine serum albumin (BSA, Rockland) in $0.02 \%$ tween 20 (SigmaAldrich) in PBS pH 7.4 (PBST) for 15 minutes at RT. After the blocking, the GST-agarose was washed twice with PBST, once with PBS and suspended into PBS. 
$250 \mu \mathrm{g}$ of anti-GST or human-IgG antibody conjugated NPs, with radioactivity of 6 and $7 \mathrm{kBq}$, respectively, were dispersed into $500 \mu \mathrm{L} 50 \%$ human plasma, diluted with PBS. Of this suspension, $200 \mu \mathrm{L}$ was added on $200 \mu \mathrm{L}$ of agarose suspension and mixed for $15 \mathrm{~min}$ at RT. The unbound particles were removed from the GST-agarose by washing three times with $800 \mu \mathrm{L}$ of PBST. The amount of GSTagarose bound particles was calculated by measuring the radioactivity of the GST-agarose with gamma counter (LKBWallac Clinigamma 1272, Wallac Oy). The samples were measured as a duplicate.

2.11. Biodistribution of O-PEGylated UnTHCPSi-NPs in Mice. All animal experiments were performed according to the guidelines approved by the Ethical Committee of the National Laboratory Animal Centre Finland (License number ESLH-2009-10004/Ym-23, Kuopio, Finland). Healthy female CD2F1 mice, weighting from 22 to 25 grams, were used in this study. The mice received chow and water ad libitum.

The animals were anaesthetized with isoflurane prior the injections. Radiolabelled and PEGylated nanoparticles (50 $\mu \mathrm{g}$ of UnTHCPSi-tyr $\left({ }^{125} \mathrm{I}\right)$-NPs PEGylated with $10 \mathrm{kDa}$ O-PEG, suspended in $100 \mu \mathrm{L}$ of PBS, pH $7.4(34 \mathrm{kBq}))$ were injected via tail vein. The mice were sacrificed immediately or ten minutes after the injections by gassing with $\mathrm{CO}_{2}(2 \mathrm{~min})$ and collecting terminal blood sample by cardiac puncture. The tissue samples, collected from the mice, were weighed and measured with gamma counter. Tissue-specific radioactivity was represented as percentage of injected dose per weight of the organ $(\% \mathrm{ID} / \mathrm{g})$. Three mice were used per one data point. The tissue samples for microscopic autoradiography and TEM were collected from liver.

2.12. Autoradiography. The tissue samples from the liver were dissected out and immersed into $4 \%$ formalin (in $0.1 \mathrm{M}$ phosphate buffer, $\mathrm{pH}$ 7.4, (PB)). After fixation for four hours, they were dehydrated in ethanol and xylene and embedded into paraffin. Using a sliding microtome, $10 \mu \mathrm{m}$ thick sections were cut on slides, deparaffinized and rehydrated. For autoradiography, the slides were dipped into emulsion $\left(+45^{\circ} \mathrm{C}\right.$, Kodak NBT Emulsion $)$ and transferred in dark and dry chamber at $+4^{\circ} \mathrm{C}$. After 1,2 , and 3 weeks, separate slides were taken out from the incubation chamber and developed with Kodal Developer D-19 for 4 min. Then they were rinsed in distilled water and fixed in Kodak Fixer for $5 \mathrm{~min}$. After rinsing in water, they were counterstained with Nuclear Fast Red for 10 min, dehydrated, cleared, and mounted with Depex and coverslip.

2.13. Electron Microscopy. For the size measurements the nanoparticles were diluted with $\mathrm{H}_{2} \mathrm{O}$ to concentrations $1.5 \mu \mathrm{g} / \mathrm{mL}$ or $0.3 \mu \mathrm{g} / \mathrm{mL}$. The diluted samples were sonicated for 5 minutes and one drop of the dilution was dried on a copper grid (mesh 200) coated with $0.3 \%$ Formvar. The samples were imaged with transmission electron microscope (TEM, JEOL JEM2100F, JEOL Ltd). The liver samples were fixed in $1 \%$ glutaraldehyde for 2 hours, then washed in
$\mathrm{PB}$ and postfixed with $1 \%$ osmium tetroxide for $1 \mathrm{~h}$. After washing in $\mathrm{PB}$, the samples were dehydrated in a series of ethanol and propylene oxide, and finally embedded into Durcupan (ACM, Fluka). After polymerization at $+65^{\circ} \mathrm{C}$, ultrathin sections were cut with Reichert Jung Ultramicrotome, stained with uranyl acetate and lead citrate. The sections were studied with TEM as described above.

\section{Results and Discussion}

3.1. Surface Modification of the UnTHCPSi-NPs. The chemically reactive carboxyl groups on the surface of UnTHCPSiNPs provide a good platform for the conjugation of biologically active molecules or tracers for biodistribution studies. Furthermore, hydrophobicity of the particles allows the adsorption of molecules, like fatty acids, directly on the surface. Initially UnTHCPSi-NPs do not form stable dispersions in physiological buffers and are thus unfavorable for in vivo studies. We modified the physicochemical properties of the particles using PEGylation either with covalent coupling (C-PEG) or adsorption via the oleyl moiety in the O-PEG conjugate.

The change of the hydrodynamic size and zeta-potential was measured after the PEGylation with DLS (Table 1) and verified with TEM (Figure 2). The TEM images showed that neither of the two PEGylation methods changed the shape of the NPs. Both PEGylation methods with molecule weights of $10 \mathrm{kDa}$ and $20 \mathrm{kDa}$ increased the size of the UnTHCPSiNPs. Interestingly, O-PEGylation of the UnTHCPSi-NPs reduced the zeta-potential more than C-PEGylation. This can be explained by steric hindrance of PEG moieties as the shear plane, where the zeta-potential is determined, is pushed further away from the particle surface by PEG [10]. Zeta-potential closer to zero millivolt indicates higher PEG coverage on the particle surface with O-PEGylation. The results are in line with the smaller increase in the size of the NPs after opsonization as well as with the finding that only O-PEGylation provided prolonged stability of the NP suspension in PBS.

3.2. In Vitro Opsonization Studies. The opsonization degrees of the UnTHCPSi-NPs with different PEGylations were tested with human plasma. When immersed in plasma, the size of the O-PEGylated particles increased less than the size of C-PEGylated or the original particles suggesting lower opsonization degree (Figure 3). The opsonization caused size highest size increment in C-PEGylated NPs. The coverage achieved with C-PEGylation is limited by the accessibility of the carboxyl groups on the NP surface. O-PEG is adsorbed overall of the particle due to the hydrophobic interaction producing better coverage than C-PEG. Due to the lower coverage rate the particles protected by C-PEGylation had a higher aggregation rate, which is shown by higher average hydrodynamic size.

In order to study the protein composition involved in the opsonization, the bound plasma proteins were separated from the PEGylated (O-PEG $10 \mathrm{kDa}$ ) and non-PEGylated nanoparticles with SDS-PAGE. The protein bands were 
TABLE 1: The hydrodynamic size, polydispersity index (PDI), and zeta-potential of the UnTHCPSi nanoparticles with different PEGylations. O-PEG is adsorbed to UnTHCPSi-NP surface while C-PEG is covalently bound with amide linkage.

\begin{tabular}{lccc}
\hline UnTHCPSi-NP PEGylation & Hydrodynamic size $(\mathrm{nm})$ & PDI & Zeta-potential $(\mathrm{mV})$ \\
\hline none & $139.6 \pm 0.7$ & $0.082 \pm 0.011$ & $-31.7 \pm 0.4$ \\
O-PEG 5k Da & $137.6 \pm 1.2$ & $0.093 \pm 0.006$ & $-28.7 \pm 0.1$ \\
O-PEG $10 \mathrm{kDa}$ & $145.3 \pm 1.2$ & $0.089 \pm 0.006$ & $-26.6 \pm 0.8$ \\
O-PEG $20 \mathrm{kDa}$ & $146.9 \pm 1.6$ & $0.079 \pm 0.017$ & $-25.6 \pm 0.3$ \\
C-PEG $5 \mathrm{kDa}$ & $134.5 \pm 1.0$ & $0.111 \pm 0.004$ & $-35.5 \pm 0.2$ \\
C-PEG $10 \mathrm{kDa}$ & $146.6 \pm 0.6$ & $0.081 \pm 0.024$ & $-32.7 \pm 0.7$ \\
C-PEG $20 \mathrm{kDa}$ & $152.8 \pm 1.5$ & $0.109 \pm 0.036$ & $-29.0 \pm 0.5$ \\
\hline
\end{tabular}

The values are represented as an average of three measurements \pm standard deviation.

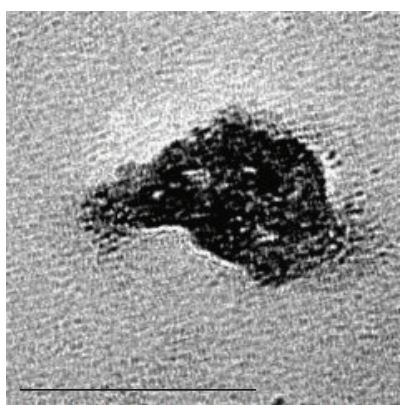

(a)

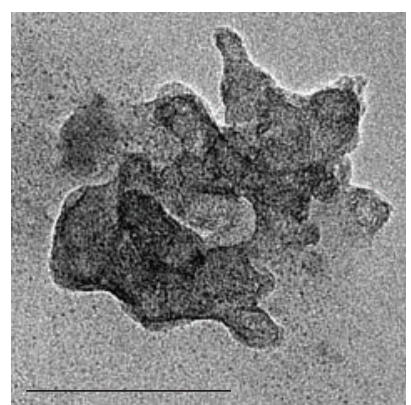

(b)

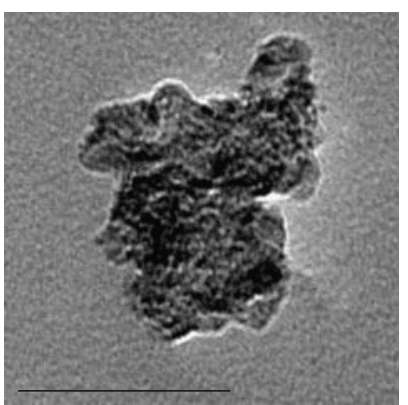

(c)

Figure 2: The TEM images from UnTHCPSi-NPs (a), $10 \mathrm{kDa}$ C-PEG UnTHCPSi-NPs (b), and $10 \mathrm{kDa}$ O-PEG UnTHCPSi-NPs (c). The length of the size bar is $100 \mathrm{~nm}$.

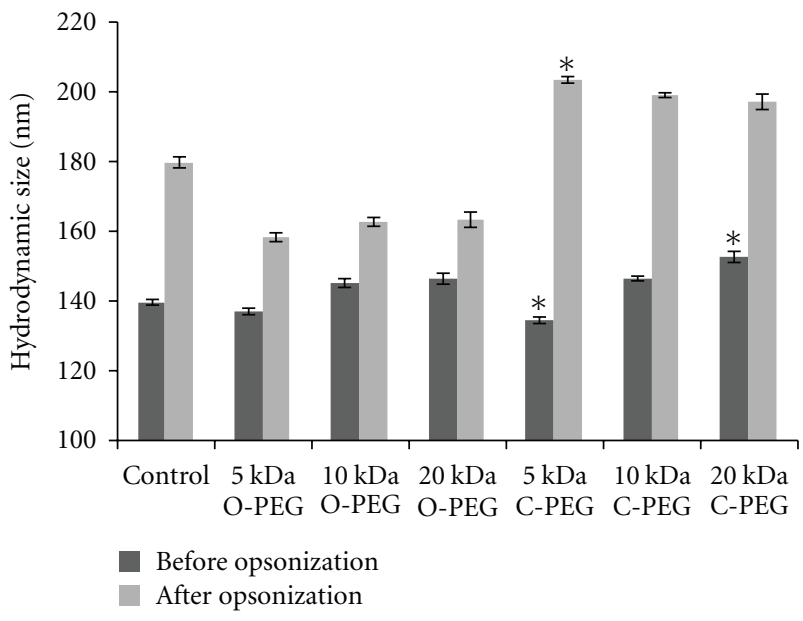

FIgURE 3: The effect of plasma immersion to the hydrodynamic size of the UnTHCPSi-NPs with two different PEGylation approaches. PEG was either adsorbed to the NP surface via hydrophobic interactions (O-PEG) or covalently bound to the carboxylic groups (C-PEG). Plain UnTHCPSi nanoparticles were used as a control. The results are presented as an average of three measurements \pm standard deviation. The PDI values $<0.10(*<0.13)$.

extracted from the gel, digested with trypsin and analyzed with mass spectrometry. The size change of the particles after the opsonization correlates with the relative intensity of the protein bands in SDS-PAGE gel. The most abundant proteins

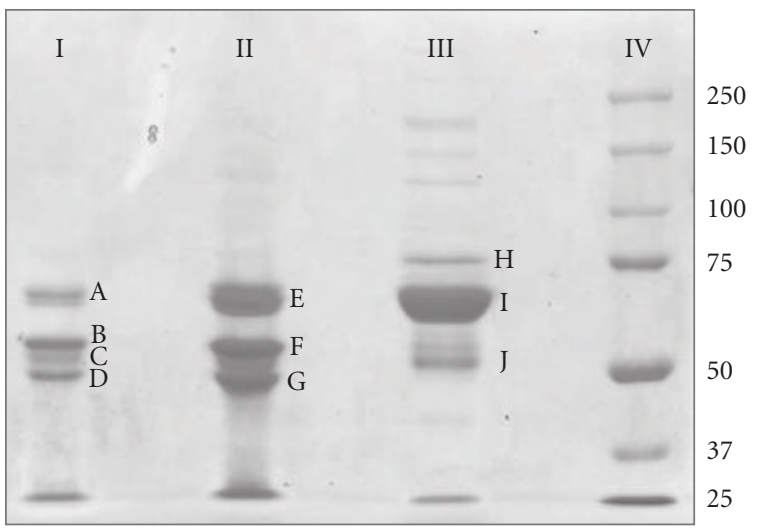

Figure 4: SDS-PAGE gel from opsonized UnTHCPSi-NPs. The bands, A-J, were excised and identified with mass spectroscopy and MASCOT search after an in-gel digestion with trypsin (Table 2). Lanes: I 10 kDa O-PEGylated UnTHCPSi-NP; II UnTHCPSi-NP; III human plasma; IV molecule weight marker.

bound on the nanoparticles were fibrinogen, Immunoglobulin G (IgG), and Human serum albumin (HSA). Fibrinogen is not the most abundant protein in plasma, but it was most abundant protein bound to the NPs. (Figure 4 and Table 2). These results are in line with previous work done by Deng et al. where fibrinogen was shown to be most abundant protein binding to negatively charged poly(acrylic acid)-coated gold nanoparticles (PAAG) from plasma. The PAAG diameter was shown to increase by $\sim 14 \mathrm{~nm}$ by 
TABLE 2: List of proteins identified from opsonized UnTHCPSi-NPs separated with SDS-PAGE. The bands correspond to the letters in Figure 3.

\begin{tabular}{llccc}
\hline Band & Proteins identified & Mw $^{\mathrm{a}}(\mathrm{Da})$ & Code $^{\mathrm{b}}$ & MASCOT score $^{\mathrm{c}}$ \\
\hline A & Fibrinogen alpha chain, isoform 2 & 69757 & P02671 & 600 \\
B & Fibrinogen beta chain & 55928 & P02675 & 548 \\
C & Ig gamma-1 chain C region & 36106 & P01857 & 180 \\
D & Fibrinogen gamma chain & 49497 & P02679 & 85 \\
E & Fibrinogen alpha chain, isoform 2 & 69757 & P02671 & 773 \\
F & Serum albumin & 69367 & P02768 & 545 \\
G & Fibrinogen beta chain & 55928 & P02675 & 950 \\
H & Fibrinogen gamma chain, isoform A & 49497 & P02679 & 275 \\
I & Serotransferrin & 77064 & P02787 & 303 \\
J & Serum albumin & 69367 & P02768 & 739 \\
\end{tabular}

${ }^{\mathrm{a}} \mathrm{Mw}$ is the nominal mass from the UniProt protein database [27]. ${ }^{\mathrm{b}}$ code is the accession number in UniProt protein database. ${ }^{\mathrm{c}} \mathrm{MASCOT}$ score is derived from the ion scores from the MS/MS search. It evaluates the reliability of the MASCOT-search.

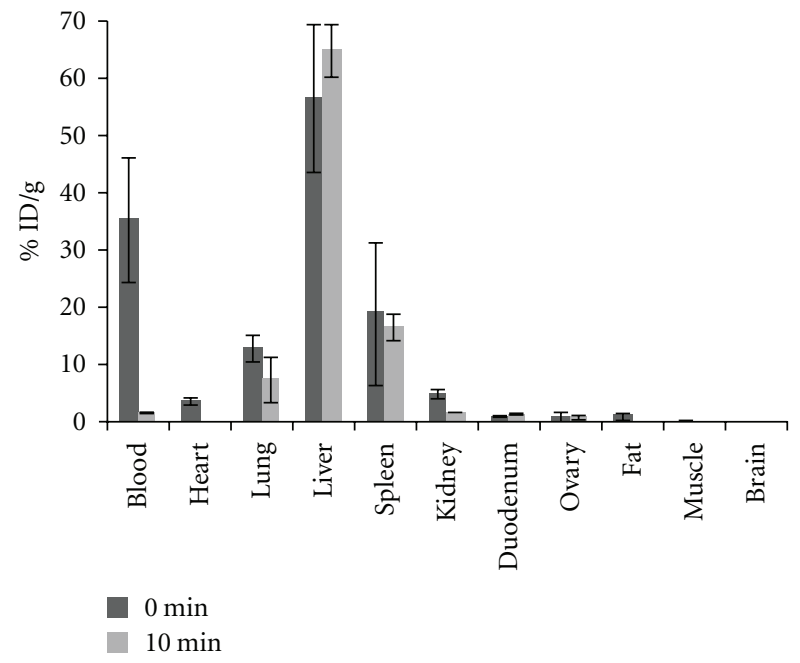

FIGURE 5: The biodistribution of the ${ }^{125} \mathrm{I}$ radiolabelled UnTHCPSiNPs with $10 \mathrm{kDa}$ O-PEGylation in healthy female mice $(n=3)$. The data is presented as a percentage of injected dose per organ weight $(\% \mathrm{ID} / \mathrm{g}) \pm$ standard deviation.

fibrinogen protein corona bound to the nanoparticle surface [26]. In our studies opsonization increased the size of OPEGylated UnTHCPSi-NPs $\sim 15 \mathrm{~nm}$, whereas the diameter of C-PEGylated NPs increased substantially more, indicating possible agglomeration of the NPs due to the insufficient PEG coverage.

3.3. Biotargeting of UnTHCPSi-NPs with Immunoprecipitation. The targeting properties of the antibody conjugated and radiolabelled UnTHCPSi-NPs were tested with GSTagarose immunoprecipitation. Anti-GST was used as the targeting antibody and human-IgG was used as a control antibody. The radiolabelled and antibody conjugated UnTHCPSi-NPs were PEGylated by adsorbing O-PEG to the nanoparticle surface and their binding affinity against
GST-agarose was tested in human plasma. Nanoparticles targeted with a-GST bound to the agarose nearly 35fold comparing to the nanoparticles conjugated with the control antibody, human-IgG. The percentages of GSTagarose bound nanoparticles were $20.8 \pm 6.2$ and $0.6 \pm$ 0.1 , respectively. This indicates that antibodies are correctly exposed on the surface of the nanoparticles and are able to target their antigen in human plasma.

3.4. Biodistribution of O-PEGylated UnTHCPSi-NPs in Mice. Since the opsonization degree was lowest with O-PEGylated UnTHCPSi particles and they were the only nanoparticles that formed stable suspension in PBS, the biodistribution of ${ }^{125}$ I-labeled and O-PEGylated (10 kDa) UnTHCPSi-NPs was studied in vivo in healthy female CD2F1 mice. The results are presented as \% of injected dose/g of tissue. The UnTHCPSi-NP circulation time in blood is short, as the radioactivity decreased from $35 \% \mathrm{ID} / \mathrm{g}$ to $2 \% \mathrm{ID} / \mathrm{g}$ within ten minutes after the injection and most of the nanoparticles accumulated to liver and spleen tissue almost instantly (Figure 5). The radiolabel appears to be stable as radioactivity in kidney was low in both time points showing minor excretion to urine.

3.5. Microscopic Autoradiography and TEM. The local biodistribution of the UnTHCPSi-NPs, PEGylated with $10 \mathrm{kDa}$ O-PEG, in liver was studied with autoradiography and transmission electron microscopy. The results revealed that the great majority of the PEGylated nanoparticles are in the lumen of the hepatic blood vessels and sinusoids after 10 minutes of the injection. However, minor part was also phagocyted by macrophages as well attached on the plasma membrane of red blood cells. Some UnTHCPSiNPs were occasionally seen internalized in membrane bound vacuoles inside the hepatocytes or attached to microvilli of the hepatocytes (Figure 6). The liver tropism is partially based on plasma proteins bound to the particles. Previously, it has been shown that the proteins bound by the 


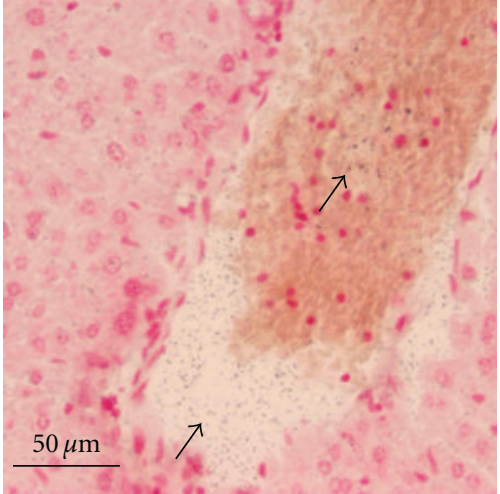

(a)

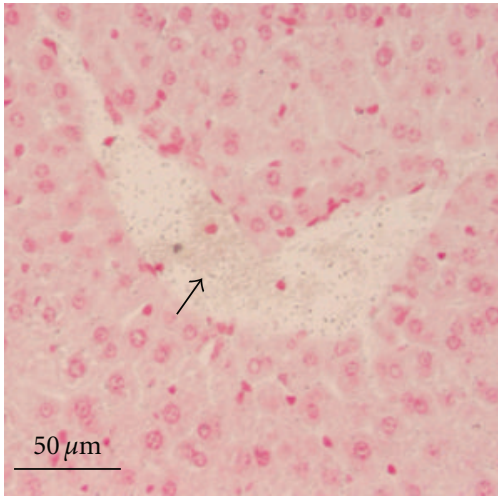

(b)

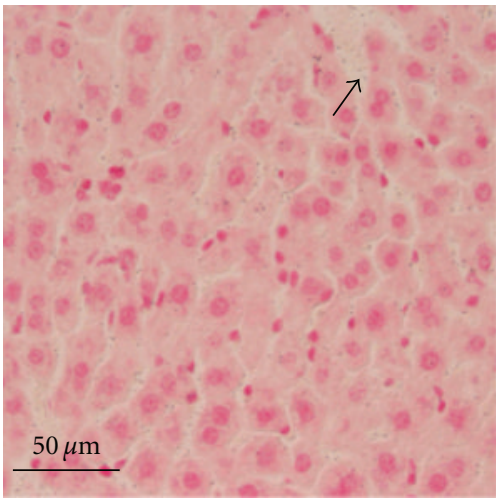

(c)

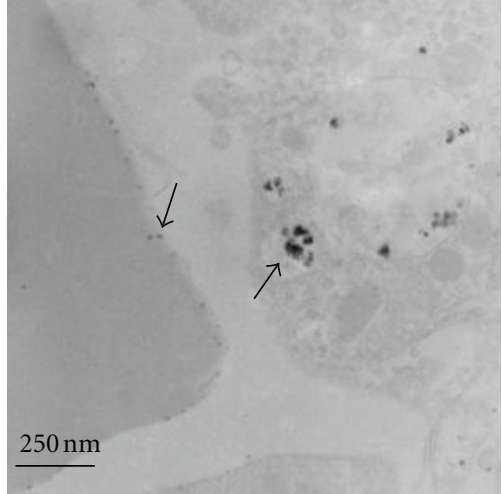

(d)

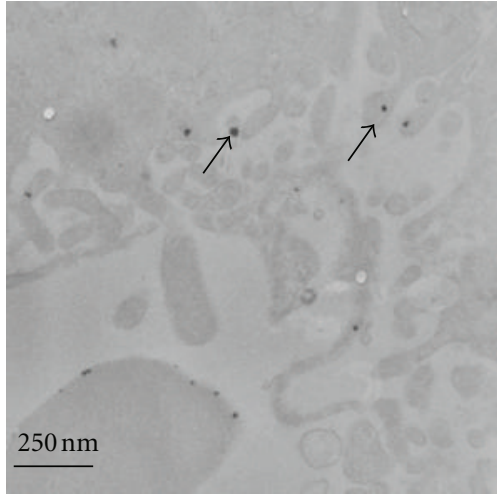

(e)

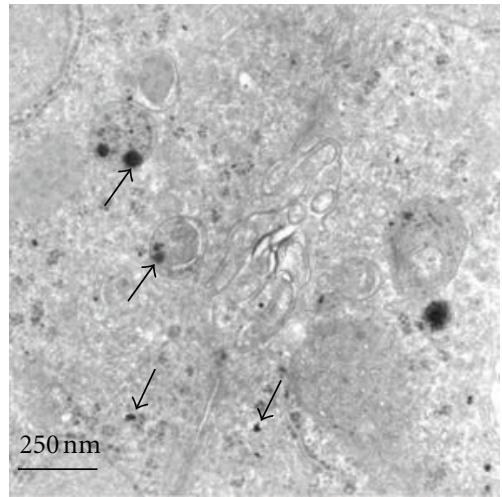

(f)

FIGURE 6: Microscopic autoradiography $(\mathrm{a}, \mathrm{b}, \mathrm{c})$ and TEM images $(\mathrm{d}, \mathrm{e}, \mathrm{f})$ from a liver of a mouse 10 minutes after the injection with $10 \mathrm{kDa}$ O-PEGylated UnTHCPSi-NPs. The nanoparticles, pointed out with black arrows, are located mainly in the lumen of liver blood vessels (a, b) and they are able to emanate into the sinusoids as well (c). However, TEM images indicate that, some of the NPs are phagocyted by macrophages (upward arrows) but are also found on the surface of the red blood cells (downward arrows) (d). Some NPs are detected in the microvilli of the liver cells (e) and in autophagocytic vacuoles inside the liver cells (upward arrows) as well as in the cytoplasm (downward arrows) (f).

nanoparticles undergo conformational changes [28-30] and thus act as immunogens by exposing buried epitopes [31]. Fibrinogen has been reported to unfold on the surface of the negatively charged poly(acrylic acid)-coated gold nanoparticles expressing buried sequences. This revealed epitope binds to the macrophage- 1 Mac-1 receptor, which express on the most abundant lymphocytes like macrophages and neutrophils $[26,32]$.

The concept of drug release from porous silicon particles, which are immobilized into the liver, has been reported previously, where intravenously administered mesoporous silicon microparticles loaded with siRNA encapsulated into nanoliposomes accumulate into the liver and spleen, but remain in the sinusoidal space enabling sustained release of siRNA loaded nanoliposomes [33]. Even though 10 minutes is a short monitoring time, our results support the previous findings. Moreover, there are some recently published results, where thermally hydrocarbonized porous silicon nanoparticles were not phagocyted in extent by RAW 264.7 macrophages in vitro. Instead, they showed a strong 
cellular association as majority of the NPs remained attached to cell membranes [18]. Furthermore, it is known that PEGylation reduces cellular interactions and internalization properties [34, 35]. Whether PEGylated UnTHCPSi-NPs can be utilized as a liver immobilized drug release platform requires further studying.

\section{Conclusions}

Here we reported the production, biofunctionalization, and targeting features of thermally carbonized porous silicon nanoparticles derivatized with undecylenic acid. These nanoparticles can be surface functionalized through the carboxyl groups, which enable conjugation of targeting moieties and tracers. The effect of two distinct PEGylation methods to UnTHCPSi-NP opsonization was studied. Our study showed that PEGylation reduced the amount of protein bound to UnTHCPSi-NPs the covalently bound PEG being less effective than the PEG adsorbed on the particles surface via hydrophobic interaction. The protein analyses revealed that the most abundant opsonin found on the UnTHCPSi-NPs was fibrinogen. Only the O-PEGylation improved the suspension stability of the UnTHCPSi-NPs in physiological buffers, which was essential for in vivo studies. Effective targeting with UnTHCPSi-NPs is possible in physiological environment, when they are conjugated with targeting antibodies. Disappointingly, the circulation time of the NPs in blood remains short, even though the PEGylation reduced the opsonization in vitro. As a conclusion, the short circulation time in vivo is the obstacle that needs to be solved in order to have targeted drug delivery in vivo with chemically modified PSi nanoparticles.

\section{Acknowledgments}

This paper has been supported by Academy of Finland (Grant no. 127138) and by the strategic funding of the University of Eastern Finland. The authors would like to thank M. Sc. Janne Weisell for his skilful assistance with mass spectrometry and proteomics.

\section{References}

[1] S. C. Bayliss, R. Heald, D. I. Fletcher, and L. D. Buckberry, "Culture of mammalian cells on nanostructured silicon," Advanced Materials, vol. 11, no. 4, pp. 318-321, 1999.

[2] J. Salonen, A. M. Kaukonen, J. Hirvonen, and V. P. Lehto, "Mesoporous silicon in drug delivery applications," Journal of Pharmaceutical Sciences, vol. 97, no. 2, pp. 632-653, 2008.

[3] T. Limnell, J. Riikonen, J. Salonen et al., "Surface chemistry and pore size affect carrier properties of mesoporous silicon microparticles," International Journal of Pharmaceutics, vol. 343, no. 1-2, pp. 141-147, 2007.

[4] D. E. Owens III and N. A. Peppas, "Opsonization, biodistribution, and pharmacokinetics of polymeric nanoparticles," International Journal of Pharmaceutics, vol. 307, no. 1, pp. 93102, 2006.

[5] M. M. Frank and L. F. Fries, "The role of complement in inflammation and phagocytosis," Immunology Today, vol. 12, no. 9, pp. 322-326, 1991.
[6] S. M. Moghimi, A. C. Hunter, and J. C. Murray, "Longcirculating and target-specific nanoparticles: theory to practice," Pharmacological Reviews, vol. 53, no. 2, pp. 283-318, 2001.

[7] Y. Geng, P. Dalhaimer, S. Cai et al., "Shape effects of filaments versus spherical particles in flow and drug delivery," Nature Nanotechnology, vol. 2, no. 4, pp. 249-255, 2007.

[8] R. B. Huang, S. Mocherla, M. J. Heslinga, P. Charoenphol, and O. Eniola-Adefeso, "Dynamic and cellular interactions of nanoparticles in vascular-targeted drug delivery (review)," Molecular Membrane Biology, vol. 27, no. 4-6, pp. 190-205, 2010.

[9] T. S. Levchenko, R. Rammohan, A. N. Lukyanov, K. R. Whiteman, and V. P. Torchilin, "Liposome clearance in mice: the effect of a separate and combined presence of surface charge and polymer coating," International Journal of Pharmaceutics, vol. 240, no. 1-2, pp. 95-102, 2002.

[10] B. Heurtault, P. Saulnier, B. Pech, J. E. Proust, and J. P. Benoit, "Physico-chemical stability of colloidal lipid particles," Biomaterials, vol. 24, no. 23, pp. 4283-4300, 2003.

[11] R. Gref, Y. Minamitake, M. T. Peracchia, V. Trubetskoy, V. Torchilin, and R. Langer, "Biodegradable long-circulating polymeric nanospheres," Science, vol. 263, no. 5153, pp. 16001603, 1994.

[12] J. Milton Harris, N. E. Martin, and M. Modi, "Pegylation: a novel process for modifying pharmacokinetics," Clinical Pharmacokinetics, vol. 40, no. 7, pp. 539-551, 2001.

[13] A. C. Faure, S. Dufort, V. Josserand et al., "Control of the in vivo biodistribution of hybrid nanoparticles with different poly(ethylene glycol) coatings," Small, vol. 5, no. 22, pp. 25652575, 2009.

[14] D. Peer, J. M. Karp, S. Hong, O. C. Farokhzad, R. Margalit, and R. Langer, "Nanocarriers as an emerging platform for cancer therapy," Nature Nanotechnology, vol. 2, no. 12, pp. 751-760, 2007.

[15] E. Ruoslahti, S. N. Bhatia, and M. J. Sailor, "Targeting of drugs and nanoparticles to tumors," Journal of Cell Biology, vol. 188, no. 6, pp. 759-768, 2010.

[16] T. Huhtala, P. Laakkonen, H. Sallinen, S. Ylä-Herttuala, and A. Närvänen, "In vivo SPECT/CT imaging of human orthotopic ovarian carcinoma xenografts with ${ }^{111}$ In-labeled monoclonal antibodies," Nuclear Medicine and Biology, vol. 37, no. 8, pp. 957-964, 2010.

[17] T. Koyama, M. Shimura, Y. Minemoto et al., "Evaluation of selective tumor detection by clinical magnetic resonance imaging using antibody-conjugated superparamagnetic iron oxide," Journal of Controlled Release, vol. 159, no. 3, pp. 413418, 2012.

[18] L. M. Bimbo, M. Sarparanta, H. A. Santos et al., "Biocompatibility of thermally hydrocarbonized porous silicon nanoparticles and their biodistribution in rats," ACS Nano, vol. 4, no. 6, pp. 3023-3032, 2010.

[19] J. Salonen, M. Björkqvist, E. Laine, and L. Niinistö, "Stabilization of porous silicon surface by thermal decomposition of acetylene," Applied Surface Science, vol. 225, no. 1-4, pp. 389394, 2004.

[20] M. Kovalainen, J. Mönkäre, E. Mäkilä et al., "Mesoporous Silicon (PSi) for sustained peptide delivery: effect of psi microparticle surface chemistry on peptide YY3-36 Release," Pharmaceutical Research, vol. 29, no. 3, pp. 837-846, 2012.

[21] L. Russo, F. Colangelo, R. Cioffi, I. Rea, and D. Stefano, "A mechanochemical approach to porous silicon nanoparticles fabrication," Materials, vol. 4, no. 6, p. 1023, 2011. 
[22] M. Silvander, N. Bergstrand, and K. Edwards, "Linkage identity is a major factor in determining the effect of PEGylated surfactants on permeability of phosphatidylcholine liposomes," Chemistry and Physics of Lipids, vol. 126, no. 1, pp. 77-83, 2003.

[23] M. Sarparanta, L. M. Bimbo, J. Rytkoänen et al., "Intravenous delivery of hydrophobin-functionalized porous silicon nanoparticles: stability, plasma protein adsorption and biodistribution," Molecular Pharmaceutics, vol. 9, no. 3, pp. 654-663, 2012.

[24] Matrix Science Ltd., "The Mascot search engine," 2010, http://www.matrixscience.com/.

[25] M. Cerrada-Gimenez, J. Häyrinen, S. Juutinen, T. Reponen, J. Jänne, and L. Alhonen, "Proteomic analysis of livers from a transgenic mouse line with activated polyamine catabolism," Amino Acids, vol. 38, no. 2, pp. 613-622, 2010.

[26] Z. J. Deng, M. Liang, M. Monteiro, I. Toth, and R. F. Minchin, "Nanoparticle-induced unfolding of fibrinogen promotes Mac-1 receptor activation and inflammation," Nature Nanotechnology, vol. 6, no. 1, pp. 39-44, 2011.

[27] UniProt Consortium, "UniProt protein database," 2011, http://www.uniprot.org/.

[28] J. H. Teichroeb, J. A. Forrest, and L. W. Jones, "Size-dependent denaturing kinetics of bovine serum albumin adsorbed onto gold nanospheres," European Physical Journal E, vol. 26, no. 4, pp. 411-415, 2008.

[29] W. Shang, J. H. Nuffer, J. S. Dordick, and R. W. Siegel, "Unfolding of ribonuclease a on silica nanoparticle surfaces," Nano Letters, vol. 7, no. 7, pp. 1991-1995, 2007.

[30] M. Lundqvist, I. Sethson, and B. H. Jonsson, "Protein adsorption onto silica nanoparticles: conformational changes depend on the particles' curvature and the protein stability," Langmuir, vol. 20, no. 24, pp. 10639-10647, 2004.

[31] I. Lynch, A. Salvati, and K. A. Dawson, "Protein-nanoparticle interactions: what does the cell see?" Nature Nanotechnology, vol. 4, no. 9, pp. 546-547, 2009.

[32] M. J. Flick, X. Du, D. P. Witte et al., "Leukocyte engagement of fibrin(ogen) via the integrin receptor $\alpha \mathrm{M} \beta 2 / \mathrm{Mac}-1$ is critical for host inflammatory response in vivo," Journal of Clinical Investigation, vol. 113, no. 11, pp. 1596-1606, 2004.

[33] T. Tanaka, L. S. Mangala, P. E. Vivas-Mejia et al., "Sustained small interfering RNA delivery by mesoporous silicon particles," Cancer Research, vol. 70, no. 9, pp. 3687-3696, 2010.

[34] N. F. Steinmetz and M. Manchester, "PEGylated viral nanoparticles for biomedicine: the impact of PEG chain length on VNP cell interactions in vitro and Ex vivo," Biomacromolecules, vol. 10, no. 4, pp. 784-792, 2009.

[35] Y. Sheng, Y. Yuan, C. Liu, X. Tao, X. Shan, and F. Xu, "In vitro macrophage uptake and in vivo biodistribution of PLA-PEG nanoparticles loaded with hemoglobin as blood substitutes: effect of PEG content," Journal of Materials Science: Materials in Medicine, vol. 20, no. 9, pp. 1881-1891, 2009. 

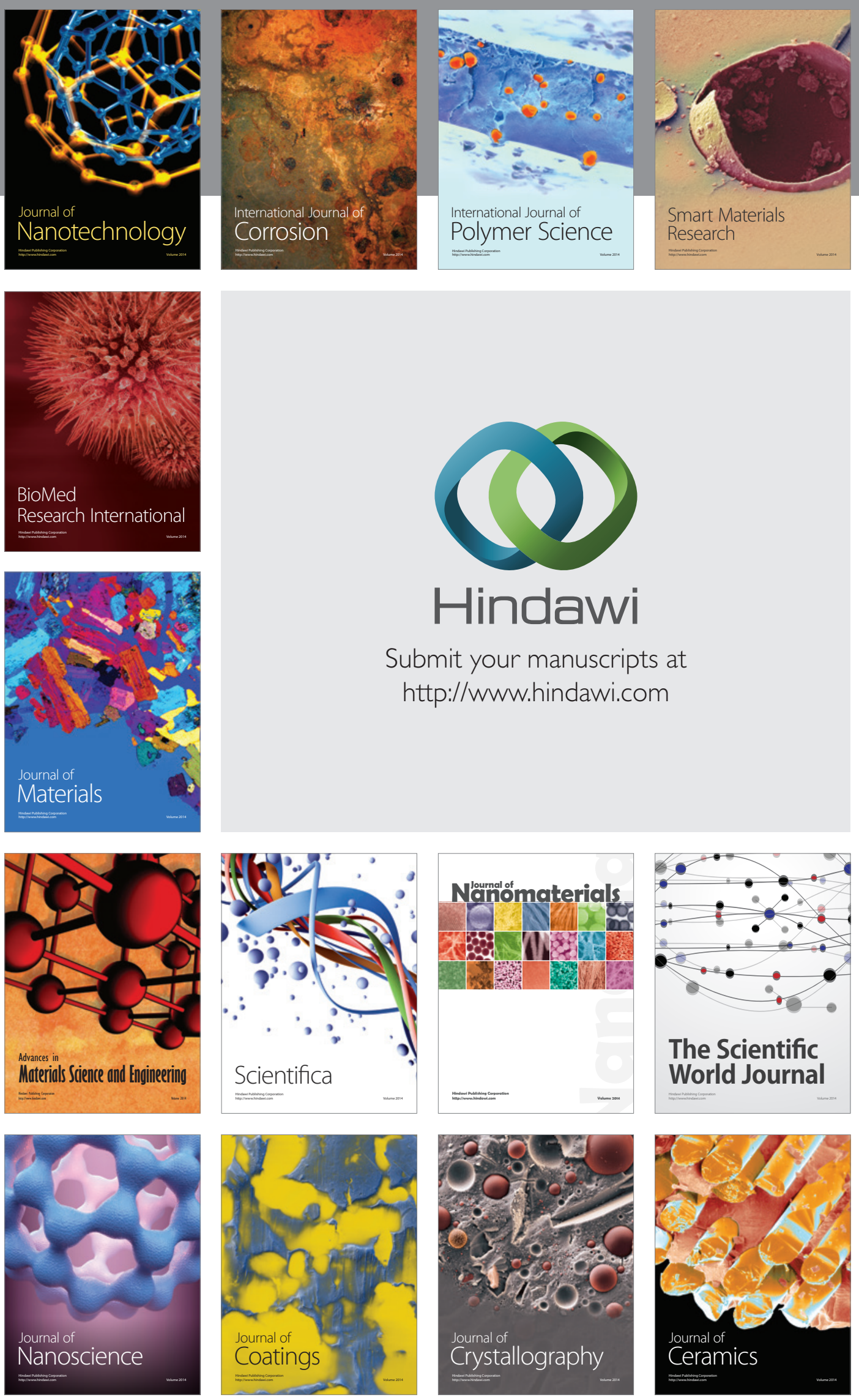

The Scientific World Journal

Submit your manuscripts at

http://www.hindawi.com

\section{World Journal}

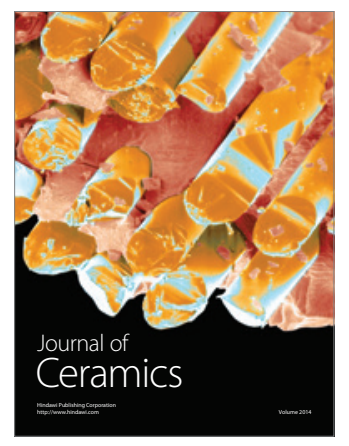

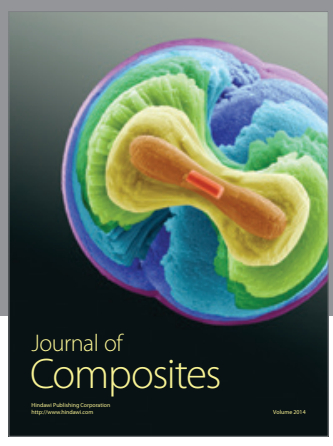
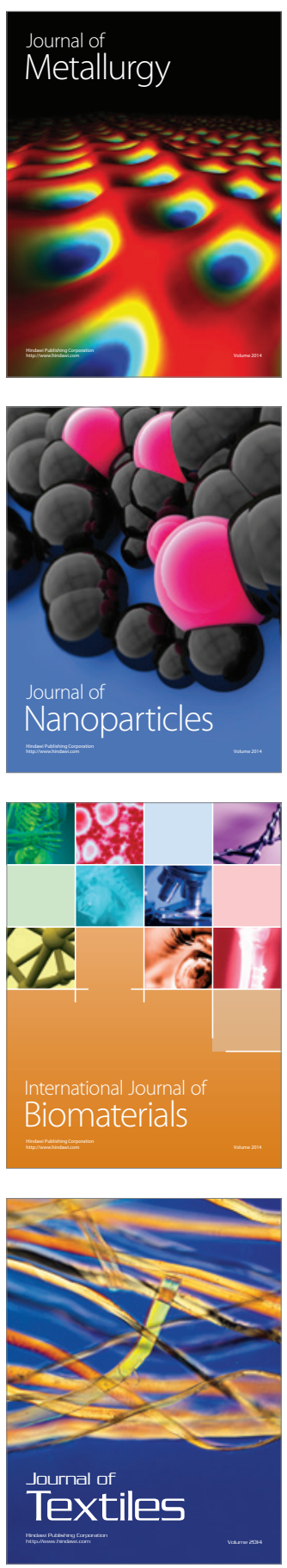